\title{
Egg quantity determination based on Characteristic Polarisation State
}

\author{
H.Ma and T.W.C Brown
}

Due to the disability of traditional Complex Natural Resonant method (CNR), the Characteristic Polarisation State (CPS) method is proposed to determine egg quantity in a box with a two by three eggs configuration. Parameters are investigated for different egg quantity scenarios and the results show a potential way to determine number in terms of characteristic angle $\beta$.

Introduction: Ultra wideband target identification is very useful in a number of applications such as military, security control, biomedical and internet of things. Intelligent fridges will become an important component of internet of things to improve quality of life. Monitoring the number of eggs in a fridge would be one application of this. As it can be known, RFID techniques can be applied to determine the rough location of an item such as a box of eggs but it cannot determine the exact quantity of eggs in the box. One possible solution is to add an RFID tag to every egg, which has disadvantages of cost and potential health risk. Therefore, UWB radar would be a useful complement to RFID in this case to determine items in a fridge and their quantity. Numerous applications on shape identification have been carried out by using the CNR method [1-4]. In these papers, people can distinguish a sphere and a cylinder or other objects well by comparing the location of independent complex natural poles. Therefore, this would provide a simple way to distinguish milk bottles and egg boxes. Nevertheless, to the authors' knowledge, there is nothing about how to determine the exact quantity. A novel method, CPS [5] has been proposed recently to observe the target polarisation. Successful story has been revealed to differ two L-shape targets with different wire length [6]. Two orthogonal linear polarisations, which are horizontal and vertical polarisation are illuminated and measured in cross and co-polarisation. Then characteristic angle in accordance to CPS is evaluated in order to have the ability to discern the number of eggs.

Determination of stable poles: Baum [7] proposed a mathematical model to represent the late time response in the early 1970 s. The non-linear waveform can be treated as a sum of exponent components with appropriate residue. The mathematical model can be expressed as follows:

$$
r(t)=\sum_{m=1}^{M} a_{m} e^{\sigma_{m}} \cos \left(\omega_{m} t+\phi_{m}\right) \quad t \geq T_{1}
$$

where $M$ is the order number, $r(t)$ is the recorded late time signal, am and $\phi_{\mathrm{m}}$ are the aspect dependent amplitude, $\sigma_{m}$ and $\omega_{m}$ are damping factor and resonant frequency respectively and pole, which can be written in the form $s_{\mathrm{m}}=\sigma_{\mathrm{m}}+\mathrm{j} \omega_{\mathrm{m}}$.

The late time can be determined as:

$T_{l}=T_{b}+T_{p}+2 T_{t r}$

where $T_{b}$ is the time when incident wave strikes the target edge, $T_{p}$ is the pulse width and $T_{t r}$ is maximum one way transmit time along the line of sight.

Scenarios of one egg in a box of two by three are under investigation. All simulations are carried in CST Microwave Studio assuming an egg box of $2 \times 3$ configuration. A plane wave source ranging from 0 to $2 \mathrm{GHz}$ is placed certain distance to the whole 
target and a probe is set far from the target to record the reflected signal. There are three possible layouts for one egg scenario due to the symmetry configuration, which can be written in the format of 100000, 001000 and 000010 (L1 R1, L2 R2, L3 R3).

The complex natural poles of one egg scenarios (Fig. 1) consist of three orders. The three orders have a trend of convergence or stable with the increase of order number. The first order with a relatively larger damping factor has been found in the left side of poles. Significant differences can be observed for different layouts. Then the second orders are mainly laid in the middle part between the first and third order. Though they are much closer to each other compared with the first order, there still exists a visible difference. While, for the third orders occurring at the right side of graph, they are quite close to each other and have a low damping factor value. Compared with the third order, first and second orders have a corresponding large damping factor which means relatively short lifetime (Lifetime is inverse proportion to the damping factor). Thus it can be summarised that the first and second poles are not fairly trustworthy due to the short lifetime and also finite solver in CST computer simulation software [8]. Therefore, the third order is highly trustworthy compared with the other two orders and can be used for further implementation of egg quantity discrimination. The property can also extend to considering the other five different quantity scenarios, where the stable value of the poles float around $-0.01 \pm 0.0295 \mathrm{j}$.

Characteristic Polarisation state: Fig. 2 illustrates us a straightforward view of extracted poles with different egg numbers. The third order poles overlapped each other, which makes CNR method impossible to ascertain the egg quantity, thus the characteristic polarisation state is introduced.

The scattered signal for target can be written as below:

$\mathbf{R}(t)=\left[\begin{array}{ll}r_{h h}(t) & r_{h v}(t) \\ r_{v h}(t) & r_{v v}(t)\end{array}\right]$

where $\mathbf{R}_{a b}(t)$ is a target matrix illuminated under polarisation state $\mathrm{b}$ and received under polarisation state $a$, which can be either horizontal or vertical. As known from [7], the reflected signal can be written as a sum of damped exponential. The parameter consists of aspect independent natural resonant poles $s_{m}=-\sigma_{m}+\mathrm{j} \omega_{m}$ and aspect dependent residues, defined by complex matrix, $\mathbf{A}_{n}$, with polarisation components as follows:

$$
\mathbf{A}_{n}=\left[\begin{array}{ll}
a_{n, h h} & a_{n, h v} \\
a_{n, v h} & a_{n, v v}
\end{array}\right]
$$

where $m$ is the order number for the complex natural poles.

According to [5], the polarisation ratio $P_{i}$ for $i=1,2,3,4$ is key parameter in determination of characteristic polarisation state and it can be deduced from above matrix. $P_{1}$ and $P_{2}$ are two maximum backscattered powers. While $P_{3}$ and $P_{4}$ are two minimum backscattered powers.

The $P_{1}$ and $P_{2}$ can be written:

$$
P_{i}=-\frac{\left|a_{n, h h}\right|^{2}+\left|a_{n, h v}\right|^{2}-\left|\gamma_{i}\right|^{2}}{a_{n, h h}^{*} a_{n, v h}+a_{n, h v}^{*} a_{n, v v}} \quad(i=1,2)
$$


where:

$\left|\gamma_{i}\right|^{2}=\frac{B}{2} \pm \frac{\sqrt{B^{2}-4 C}}{2}$

$B=\left|a_{n, h h}\right|^{2}+2\left|a_{n, h v}\right|^{2}+\left|a_{n, v v}\right|^{2}$

$C=\left|a_{n, h h} a_{n, v v}\right|^{2}+\left|a_{n, h v}\right|^{4}-2 \operatorname{Re}\left(a_{n, h h}\left(a_{n, h v}^{*}\right)^{2} a_{n, v v}\right)$

$P_{3}$ and $P_{4}$ can be written as:

$P_{3,4}= \pm \frac{r \pm a_{n, h v}}{a_{n, v v}}$

where $r=\sqrt{a_{n, h v}^{2}-a_{n, h h} a_{n, v v}}$.

The Stoke parameters can be obtained in terms of each power. The ellipticity $\varepsilon$, title angle $\tau$ and characteristic angle $\beta$ are components of stoke parameters. The extracted three parameters related with different egg quantities are also obtained.

$\varepsilon=\frac{1}{2} \sin ^{-1}\left(\frac{G_{3}}{G_{0}}\right) \quad \tau=\frac{1}{2} \tan ^{-1}\left(\frac{G_{2}}{G_{1}}\right) \quad \tan ^{2} \beta=\frac{\left|\gamma_{2}\right|}{\left|\gamma_{1}\right|}$

where $G_{0}, G_{1}, G_{2}$ and $G_{3}$ are basic element in stoke vector and can be given as:

$\frac{G_{1}}{G_{0}}=\frac{1-\left|P_{n}\right|^{2}}{1+\left|P_{n}\right|^{2}} \quad \frac{G_{2}}{G_{0}}=\frac{2 \operatorname{Re}\left(P_{n}\right)}{1+\left|P_{n}\right|^{2}} \quad \frac{G_{3}}{G_{0}}=\frac{2 \operatorname{Im}\left(P_{n}\right)}{1+\left|P_{n}\right|^{2}}$

where $n$ is an integer valid from 1 to 4 . However, the value of $\beta$ does not depend on $P_{n}$.

Numerical result: The relationship between characteristic angle $\beta$ and egg quantity are revealed in Fig. 3. The characteristic angle expresses target polarisability property. It can be found that the angle decreases with the egg number increases from above figure. The larger quantity with more complex configuration is more prone to polarized wave due to the small value $\beta$. And the result also validate that in [5] which is larger $\beta$ values are less able to polarize waves than those with smaller $\beta$ values.

Conclusion: The CPS method has been introduced and investigated. Compared with traditional CNR method, it performs a more powerful way in the target identifications. The characteristic angle is inverse proportional to egg number in terms of CPS method. Therefore, it provides a potential way to implement such results in a neural network for classification. 


\section{References}

1 L, ZHANG., Y. HAO., and C.G.PARINI. : 'Natural resonance frequency extraction for concealed weapon detection at millimetre wave frequencies', European Conference of Antennas and Propagation, 2007, Edinburgh.

2 Hoi-Shun Lui., Nicholas Shuley., and Stuart Ceozier. : 'A concept for hip prosthesis identification using Ultra Wideband Radar', IEEE International Conference in Medicine Engineering and Biology, 2004, San Francisco.

3 C.O.Hargrave., N.V.Shuley., M.E.Bialkowski., and J.C.Ralston. : 'Radar target identification of mining infrastructure for automated mine machinery navigation', European Conference of Antennas and Propagation, 2011, Rome.

4 A.Mroue., M.Heddebaut., F.Elbahhar., A.Rivenq., and J.M.Rouvaen. : 'UWB radar and leak waveguide for fall on track object identification', IEEE Radar Conf., 2010, Washington, DC.

5 H.Mott. : 'Remote sensing with polarmetric radar', Wiley, 2007.

6 Hoi-Shun Lui., and Mikael Persson. : 'Characterization of radar targets based on ultra wideband polarimetric transient signatures', General Assembly and Scientific Symposium., 2011, Istanbul.

7 Baum, C.E., Rothwell, E.J., Chen, K.M., and Nyquist, D.P. : 'The singularity expansion method and its application to target identification', Proc. IEEE, 1991, Vol. 79 , Issue 10, pp.1481-1492.

8 S.W. Harmer., D.A. Andrews., N.D.Rezgui., and N.J. Bowring. : 'Detection of handguns by their complex natural resonant frequencies', IET Microwave Antennas Propagation., 2010, Vol.4, Issue 9, pp. 1182-1190.

\section{Authors' affiliations:}

H.MA and T.W.C. Brown (Centre for Communication Research Centre, University of Surrey, Guildford GU2 7XH, United Kingdom) 
Figure captions:

Fig.1 Extracted CNR for all one egg scenarios

- $100000 \Delta 001000 \bullet 000010$

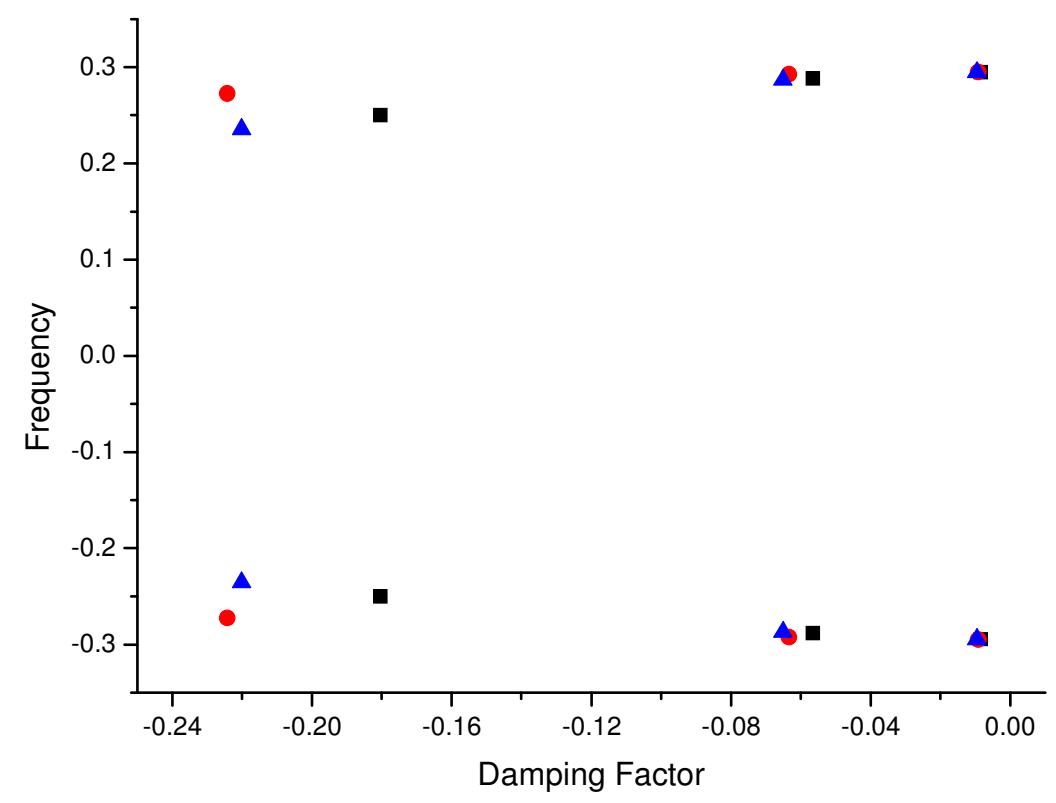

Fig.2 Extracted Third Poles for All Cases

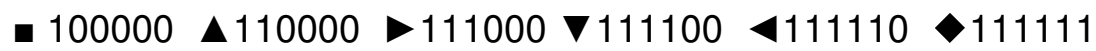

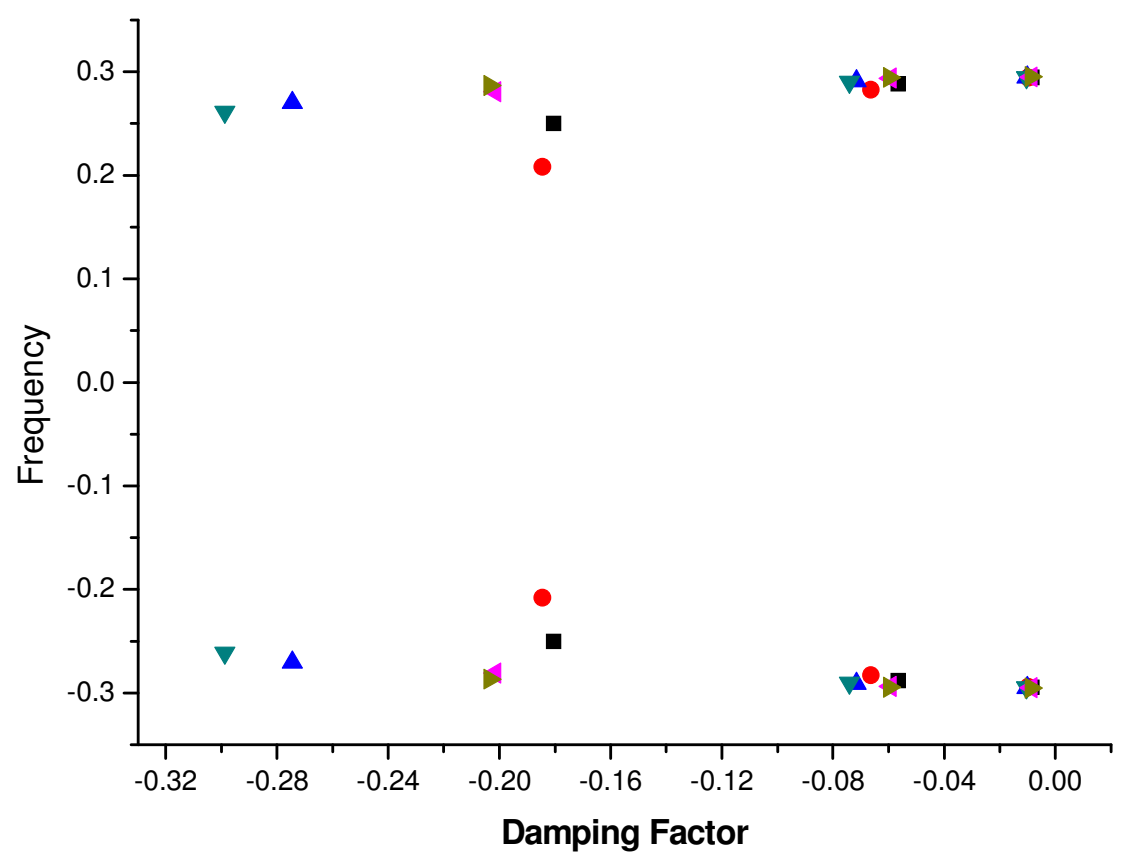


Figure 3 - Relationship between Quantity and Characteristic Angle $\beta$

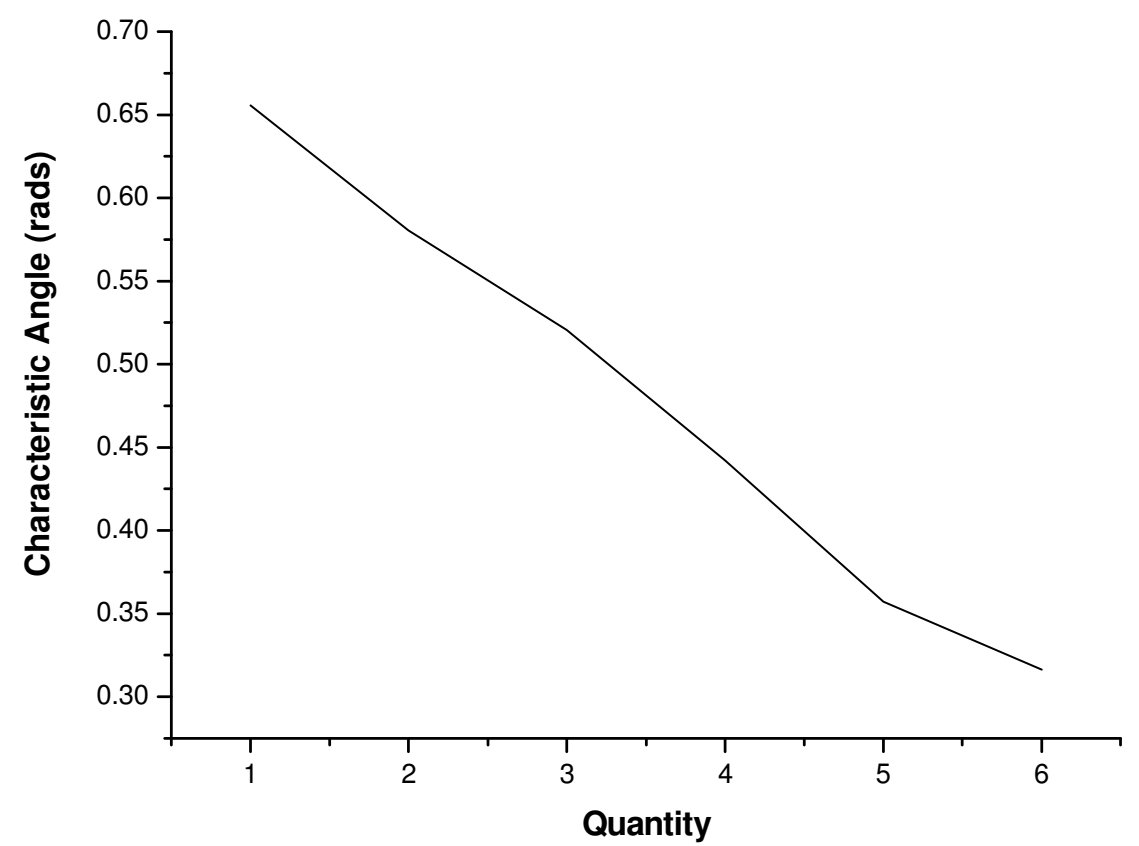

